\title{
SPECTRAL-DOMAIN MODELLING OF SUPERCONDUCTING MICROSTRIP STRUCTURES
}

\author{
SMAIN AMARI* AND JENS BORNEMANN \\ Laboratory for Lightwave Electronics, Microwaves and Communications, LLiMiC, Department of Electrical and \\ Computer Engineering, University of Victoria, Victoria B.C., Canada V8W 3P6
}

\begin{abstract}
SUMMARY
We present an analysis of thin microstrip superconducting structures in which the effects of the superconducting material, of finite complex conductivity and finite thickness, are taken into account through the concept of sheet impedance coupled with an extension of the Spectral Domain Immittance Approach. The effect of the magnetic field on the penetration depth in the superconductor is included by solving the Ginzburg-Landau equations within the thin-film approximation $\left(t / \lambda_{0} \ll 1\right)$. The state of the material is determined self-consistently in such a way that when the critical field is exceeded, the material systematically shifts from the superconducting to the normal state. Special attention is paid to the behaviour of the system in the neighbourhood of the critical temperature, $T / T_{\mathrm{c}} \simeq 1$. We report that, in some cases, the propagation constant of a superconducting microstrip line attains a maximum when the real and imaginary parts of the conductivity are approximately equal. Numerical results for a microstrip line and a patch resonator are presented and compared with available data to document the validity of the approach. (C) 1997 by John Wiley \& Sons, Ltd.
\end{abstract}

Int. J. Numer. Model., 10, 217-229 (1997)

No. of Figures: 9. No. of Tables: 0. No. of References: 18.

\section{INTRODUCTION}

The inductive properties of superconductors, in addition to their exceedingly small ohmic losses, make superconducting thin films ideal for low-loss, low-dispersion transmission lines, interconnects and microwave filters. Also, the recent discovery of high-temperature superconductors has reduced the demands on bulky and costly cryogenics required to cool the system below already low critical temperatures. Despite these advances, accurate computer models are not only desirable but are rather necessary for any efficient investigation of the properties of superconducting devices for microwave applications. Cost-effective and accurate design and analysis of superconducting microwave devices rely on adequate modelling of the electrodynamic properties of the superconducting material.

The phase velocities and attenuation constants of very thin superconducting strips were analysed by Pond and co-workers using a full-wave analysis consisting in a combination of the spectraldomain approach and sheet resistance. ${ }^{1}$ In this approach, the Green impedance dyadics are modified to include diagonal metallic terms which are equal to the sheet resistance $R=\frac{1}{\sigma t}$. This method was also applied to analyse a superconducting microstrip line using pulse functions. ${ }^{2}$ Similarly, resonant frequencies and field patterns of microstrip antennas on anisotropic substrates were determined using the same approach for both conventional and superconducting materials. ${ }^{3}$ Nghiem et al. took into consideration the unequal distribution of the surface current density on the two sides of the conductor to modify the surface (sheet) impedance in the modified Green impedance dyadics. ${ }^{4}$ The traditional surface impedance was replaced by an effective surface impedance which guarantees power conservation.

*Correspondence to: S. Amari, Department of Electrical and Computer Engineering, University of Victoria, P.O. Box 3055, Victoria B.C., Canada V8W 3P6 
In all these papers, the surface, or sheet impedance, is assumed independent of the field distribution in the structure; it depends only on the thickness and conductivity of the superconducting strip. Consequently, only diagonal metallic matrix elements appear in the modified Green impedance dyadics. The coupling of the LSE and LSM modes, however, lead to non-diagonal metallic elements especially for thin conductors, when the electromagnetic field varies either rapidly or very slowly in the direction orthogonal to the film. ${ }^{5}$ For thick films, both the surface and the sheet impedances reduce to the usual surface impedance regardless of the field properties.

A quasi-TEM analysis which includes the effect of the magnetic field on the penetration depth into the superconductor was presented in Reference 6 and applied to determine the properties of a wide microstrip line on top of a thin substrate. The solution to the Ginzburg-Landau (GL) equation was used to determine the critical fields ${ }^{6}$ and powers assuming that the magnetic field is tangential to the superconductor. The complex conductivity was modified to account for the effect of the magnetic field. A similar result was recently presented by Megahed and El-Ghazaly where the non-linear effects in the superconductor are also included. ${ }^{7}$ The GL equations were solved in the time domain using the finite-difference time-domain method (FD-TD) to handle the non-linear equations. They reported that the superconducting state is first destroyed at the edges of thin microstrip lines where the current exceeds the critical value $J_{c}$. This rather intuitive result is expected to hold qualitatively but its quantitative value is limited since the validity of the GL equations when the vector potential varies rapidly in space, as it does at the edges of a thin microstrip, is questionable. ${ }^{8}$ Under those conditions, higher-order terms in the free energy expansion become important and may not be ignored. The equations which would result are of a higher order of non-linearity and too complicated to be of much practical value in engineering design.

In this paper, we also include the effect of the magnetic field and determine the penetration depth when the superconducting strip is carrying a current density. The imaginary part of the complex conductivity obtained from the two-fluid model of Gorter and Cazimir is modified to include the effect of the magnetic field through the modification of the penetration depth following the analysis of Douglas. ${ }^{9}$ Conservation of charge then requires the real part of the conductivity to be changed, as will be discussed later.

It has been recently proposed by $\mathrm{Ma}$ and Wolff that the existence of scattering of super- as well as normal electrons in the two-fluid model leads to modified London equations. ${ }^{10}$ They report that the enhanced peaks in the real part of the complex conductivity of some high-temperature superconductors, ${ }^{11}$ observed below the critical temperature, can be explained within their model. ${ }^{10}$ In this work, we limit the analysis to the local approximation where the scattering of superelectrons is neglected.

This paper is organized as follows. In Section 2, the main points of the two-fluid model are briefly reviewed. Section 3 investigates the effect of a tangential magnetic field on the penetration depth and electric conductivity of a thin superconducting film. Section 4 shows typical numerical results obtained from the present approach and compares them to previously published results.

\section{THE TWO-FLUID MODEL}

The two-fluid model, proposed by Gorter and Cazimir, separates the electrons in the superconductor into two dynamically distinct groups: normal electrons, which undergo scattering from atoms and impurities as well as one another, and superconducting electrons, which move under the action of the electric field insensitive to any scattering mechanism. The normal component of the current depends on the electric field in accordance with Ohm's law, i.e. ${ }^{12}$

$$
\mathbf{J}_{\mathrm{n}}=\sigma_{1} \mathbf{E}
$$

where the conductivity $\sigma_{1}$ is assumed to be real. On the other hand, the superconducting part is related to the electric field by the London equation

$$
\frac{\delta \mathbf{J}_{\mathrm{s}}}{\delta t}=\frac{\mathbf{E}}{\mu_{0} \lambda^{2}}
$$

where $\lambda$ is the penetration depth. For a time-harmonic field, the first Maxwell equation then becomes 


$$
\nabla \times \mathbf{H}=\left(\sigma_{1}-\mathrm{j} \sigma_{2}\right) \mathbf{E}+\mathrm{j} \omega \epsilon \mathbf{E}
$$

where $\sigma_{2}=\frac{1}{\omega \mu_{0} \lambda^{2}}$. Hence, the superconductor is treated as a material with complex conductivity, $\sigma=\sigma_{1}-\mathrm{j} \sigma_{2}$.

Within this model, the conductivity varies with temperature according to the expressions

$$
\sigma_{1}=\sigma_{\mathrm{n}}\left(\frac{T}{T_{\mathrm{c}}}\right)^{4}
$$

and

$$
\sigma_{2}=\frac{1-\left(\frac{T}{T_{\mathrm{c}}}\right)^{4}}{\omega \mu_{0} \lambda_{0}^{2}}
$$

Note that the two-fluid model is local and applies to uniform systems where the density of superelectrons is position-independent and unaffected by the magnetic field. The phenomenological theory advanced by Ginzburg and Landau not only includes spatial dependence of the order parameter but describes the effect of a magnetic field on the superconducting state as well. The derivation of these equations is not presented here; the reader is referred to the numerous books on the subject, e.g. References 13-15.

\section{DEPENDENCE OF CONDUCTIVITY ON MAGNETIC FIELD}

It is well-established that a strong enough magnetic field destroys superconductivity and causes a phase transition to the normal state. ${ }^{13-15}$ The material-dependent threshold value of the magnetic field at which such a transition takes place depends on the temperature, the geometry and dimensions of the superconductor as well as its impurity concentration and the nature of the applied field. The magnetic field in the superconductor acts as a pair-breaking mechanism leading to an increase in the penetration depth with the strength of the magnetic field. In this paper, we consider only the effect of a magnetic field which is parallel to the surface of the superconductor. This approximation is, judging from the results presented in Reference 6, adequate.

A detailed investigation of the dependence of the penetration depth on the magnetic field was presented by Douglas, who solved the Ginzburg-Landau equations when a tangential magnetic field is applied to a thin film. ${ }^{9}$ When the thickness of the film is much smaller than the penetration depth, the order parameter can be taken as a constant. Under these conditions, the penetration depth $\lambda(T, H)$ is related to the order parameter in the Ginzburg-Landau theory by ${ }^{9}$

$$
\frac{\lambda(H, T)}{\lambda(0, T)}=\frac{\Psi(0, T)}{\Psi(H, T)}
$$

The determination of $\lambda(H, T)$ is therefore reduced to solving the Ginzburg-Landau equations with the appropriate boundary conditions.

We consider the case of a magnetic field parallel to the thin film as shown in Figure 1. The

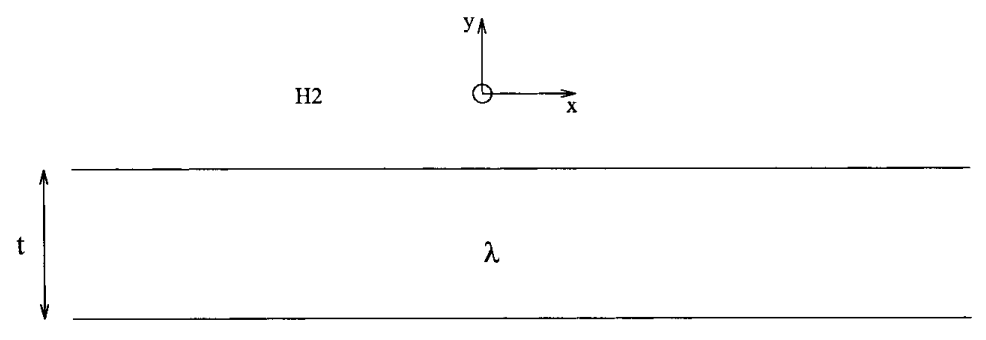

$\mathrm{H} 1$

Figure 1. Thin superconducting film, $t / \lambda \ll 1$, in a tangential magnetic field. The field is equal to $H_{1}$ on one side and $H_{2}$ on the other 
system of axes is chosen such that the magnetic field is $\mathbf{H}=H_{z} \mathbf{u}_{\mathbf{z}}$. In this analysis, we assume that the magnetic field depends only on $y$. This assumption is reasonable for wide lines and patches. The vector potential can then be written as

$$
a_{x}=\frac{h_{2}-h_{1} \cosh \left[\frac{\phi t}{\lambda(T, 0)}\right]}{\phi \sinh \left[\frac{\phi t}{\lambda(T, 0)}\right]} \cosh (\phi \eta)+\frac{h_{1}}{\phi} \sinh (\phi \eta)
$$

where $h_{1}$ and $h_{2}$ are the values of the magnetic field at the top and bottom surfaces of the film.

The Ginzburg-Landau equations, which govern both the order parameter and the applied magnetic field, can be written as

$$
\begin{aligned}
\frac{\partial^{2} a_{x}}{\partial \eta^{2}} & =\phi^{2} a_{x} \\
\frac{\partial^{2} \phi}{\partial \eta^{2}} & =\phi \kappa^{2}\left(a_{x}^{2}+\phi^{2}-1\right) \\
\frac{\partial \phi}{\partial \eta} & =0
\end{aligned}
$$

at the surface, where

$$
\begin{aligned}
& \phi=\frac{\Psi(T, H)}{\Psi(T, 0)}, \mathbf{a}=\frac{\mathbf{A}}{\sqrt{2 \lambda(T, 0) H_{\mathrm{cb}}(T)}} \\
& \eta=\frac{y}{\lambda(T, 0)}, \mathbf{h}=\frac{\mathbf{H}}{\sqrt{2 H_{\mathrm{cb}}(T)}}
\end{aligned}
$$

$\Psi$ is the order parameter, A the vector potential, $\lambda$ the penetration depth, $\kappa$ the Ginzburg-Landau parameter and $H_{\mathrm{cb}}(T)$ the bulk critical field. The boundary condition, equation $(8 b)$, corresponds to vanishing current in the direction normal to the film at the dielectric-superconductor interface. Using the assumption that $\phi$ is constant in equations (7) and (8a), using the boundary condition $(8 b)$ and integrating both sides of equation $(8 a)$, we get

$$
1-\phi^{2}=\frac{\lambda(T, 0)}{t} \int_{0}^{\frac{t}{\lambda(T, 0)}} a_{y}^{2} \mathrm{~d} \eta
$$

Combining equations (6) and (9) yields

$$
\begin{aligned}
1-\phi^{2} & =\frac{\lambda(T, 0)}{2 t \phi^{3} \sinh ^{2}[\phi t / \lambda(T, 0)]}\left[\left(h_{1}-h_{2}\right)^{2} \cosh ^{2}\left(\frac{\phi t}{2 \lambda(T, 0)}\right)\left[\sinh \left(\frac{\phi t}{\lambda(T, 0)}\right)+\frac{\phi t}{\lambda(T, 0)}\right]\right. \\
& \left.+\left(h_{1}+h_{2}\right)^{2} \sinh ^{2}\left(\frac{\phi t}{2 \lambda(T, 0)}\right)\left[\sinh \left(\frac{\phi t}{\lambda(T, 0)}\right)-\frac{\phi t}{\lambda(T, 0)}\right]\right]
\end{aligned}
$$

At a given temperature, equation (10) is solved for the quantity $\phi$. Figure 2 shows the dependence of the left- and right-hand sides as $\phi$ is varied from zero (normal state) to 1 (superconducting state). The smaller solution is rejected on the ground that it approaches zero as the current in the film vanishes.

In the structures under consideration, the thin films are carrying electric currents. From Maxwell's equations it follows that the term containing the difference $h_{2}-h_{1}=J$ dominates the right-handside of equation (6). This approximation is even more accurate for thin films, i.e. $t / \lambda(T, 0) \ll 1$. Under these conditions, convenient expressions for the normalized order parameter can be obtained 


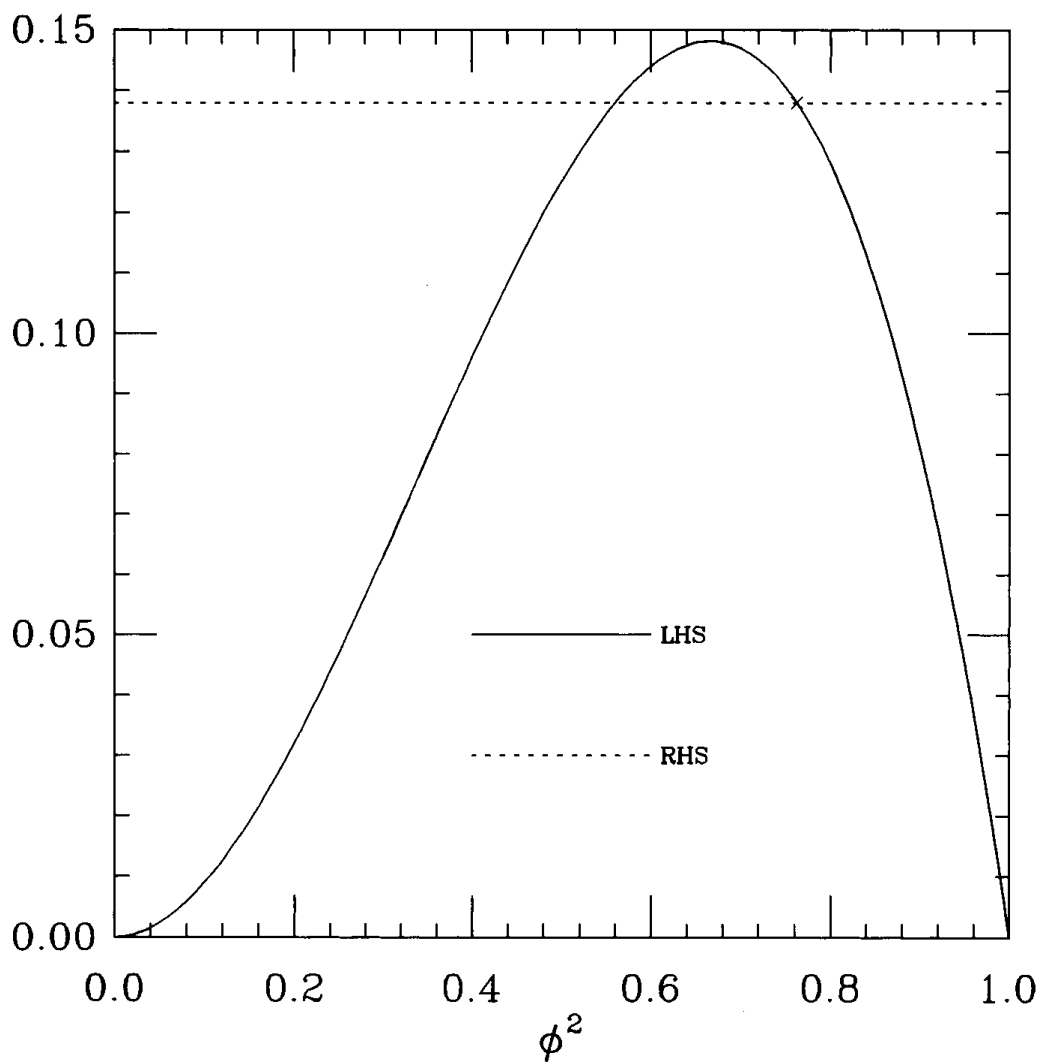

Figure 2. Solution of the Ginzburg-Landau equation. The smaller solution is rejected since it gives a normal state in zero magnetic field

from a Taylor expansion of the hyperbolic functions in equation (10) in powers of $t / \lambda(T, 0)$, leading to

$$
1-\phi^{2}=\left[\frac{\lambda(T, 0)}{t}\right]^{2} \frac{\left(h_{1}-h_{2}\right)^{2}}{\phi^{4}}
$$

If it is further assumed that the electric current in the film is not too large, $\left|H_{1}-H_{2}\right| \ll H_{\mathrm{cb}}(T)$, then

$$
\phi^{2}=1-\frac{1}{2}\left[\frac{\lambda(T, 0)}{t}\right]^{2}\left[\frac{H_{2}-H_{1}}{H_{\mathrm{cb}}(T)}\right]^{2}
$$

Once the order parameter is determined, a complex conductivity which takes into account charge conservation may be written as

$$
\begin{aligned}
& \sigma_{1}=\sigma_{\mathrm{n}}\left(\frac{T}{T_{\mathrm{c}}}\right)^{4} \phi^{2}+\sigma_{\mathrm{n}}\left(1-\phi^{2}\right) \\
& \sigma_{2}=\frac{1-\left(\frac{T}{T_{\mathrm{c}}}\right)^{4}}{\omega \mu_{0} \lambda^{2}(0,0)} \phi^{2}
\end{aligned}
$$

Similar expressions were given in References 6 and 7. If the penetration depth in zero magnetic field, $\lambda(T, 0)$, and the bulk critical magnetic field, $H_{\mathrm{cb}}(T)$, are assumed to depend on temperature according to the experimental expressions

$$
H_{\mathrm{cb}}(T)=H_{\mathrm{cb}}(0)\left[1-\left(\frac{T}{T_{\mathrm{c}}}\right)^{2}\right]
$$




$$
\lambda(T, 0)=\frac{\lambda(0,0)}{1-\left(\frac{T}{T_{\mathrm{c}}}\right)^{4}}
$$

then equations (13) can be reduced further, when the applied magnetic field is weak, leading to

$$
\begin{gathered}
\sigma_{1}=\sigma_{\mathrm{n}}\left(\frac{T}{T_{\mathrm{c}}}\right)^{4}+\sigma_{\mathrm{n}} \frac{\left(\frac{\lambda(0,0)}{t}\right)^{2}\left(\frac{I}{I_{\mathrm{c}}}\right)^{2}}{1-\left(\frac{T}{T_{\mathrm{c}}}\right)^{2}} \\
\sigma_{2}=\frac{1-\left(\frac{T}{T_{\mathrm{c}}}\right)^{4}}{\omega \mu_{0} \lambda^{2}(0,0)}-\frac{\left(\frac{I}{I_{\mathrm{c}}}\right)^{2}}{\omega \mu_{0} t^{2}\left[1-\left(\frac{T}{T_{\mathrm{c}}}\right)^{2}\right]}
\end{gathered}
$$

Note that these equations reduce to those of the two-fluid model in the proper limits. The quantity $I_{\mathrm{c}}$ is related to the bulk critical magnetic current by $w H_{\mathrm{cb}}(T=0)=I_{\mathrm{c}}$, where $w$ is the width of the strip and $I$ is the current carried by the strip.

Once a conductivity is given, the effect of the thin superconductor on the propagation or resonant properties of the structure enter our formulation through the concept of sheet impedance, as discussed in Reference 16.

\section{RESULTS}

\subsection{Microstrip}

The approach described here is first applied to a single thin superconducting microstrip line with a superconducting ground plane. The strip is $25 \mu \mathrm{m}$ wide, and the dielectric is $450 \AA$ thick with $\epsilon_{\mathrm{r}}=10 \cdot 5$. The penetration depth of the superconductor at $T=0$ is taken to be $3200 \AA$ and its normal conductivity $\sigma_{\mathrm{n}}=10^{6} \mathrm{~S} / \mathrm{m}$.

Figure 3 shows the normalized propagation constant as a function of $T / T_{\mathrm{c}}$ at $1 \mathrm{GHz}$. We first compare our results with those presented in Reference 1, where a strip line having its ground plane width equal to its conductor width is simulated instead of the original structure. The solid line in Figure 3 is obtained from the present approach, where the LSE and LSM sheet impedances are not necessarily equal, while the dashed line corresponds to both LSE and LSM sheet impedances equal to $R=1 / \sigma t$. The squares and the experimental results are from Reference 1 . Note that using different LSE and LSM sheet impedances brings the numerical results in better agreement with the measured values. If the original structure, which includes a full ground plane, instead of only one of width $w$ used in Reference 1, is analysed, lower values of the normalized propagation constant are obtained (long-dashed line). This is expected since the current is no longer confined to the cross-section of the strip line, thereby reducing the inductance and the propagation constant. Taking into consideration the fact that measured values of $\lambda_{\mathrm{L}}$ have limited accuracy, the experimental values of the propagation constant may be reproduced with a larger value of the London penetration depth. This is shown in Figure 4, where it can be seen that an increase in $\lambda_{\mathrm{L}}$ leads to an increase in the propagation constant. In other words, a better fit is obtained with a larger value of $\lambda_{\mathrm{L}}$ within this model.

We also investigated the effect of changing the normal conductivity $\sigma_{\mathrm{n}}$ on the propagation characteristics of the same structure. For large values of $\sigma_{n}$, a peak appears in the propagation constant just below the critical temperature as seen in Figure 5. This behaviour has been reported by Swihart for the case of a strip line where the phase velocity shows a minimum as the increasing temperature approaches $T_{\mathrm{c}}{ }^{17}$ As the conductivity is further decreased, the peak in the propagation constant becomes less pronounced and disappears at $\sigma_{\mathrm{n}}=10^{6} \mathrm{~S} / \mathrm{m}$ for the present structure. 


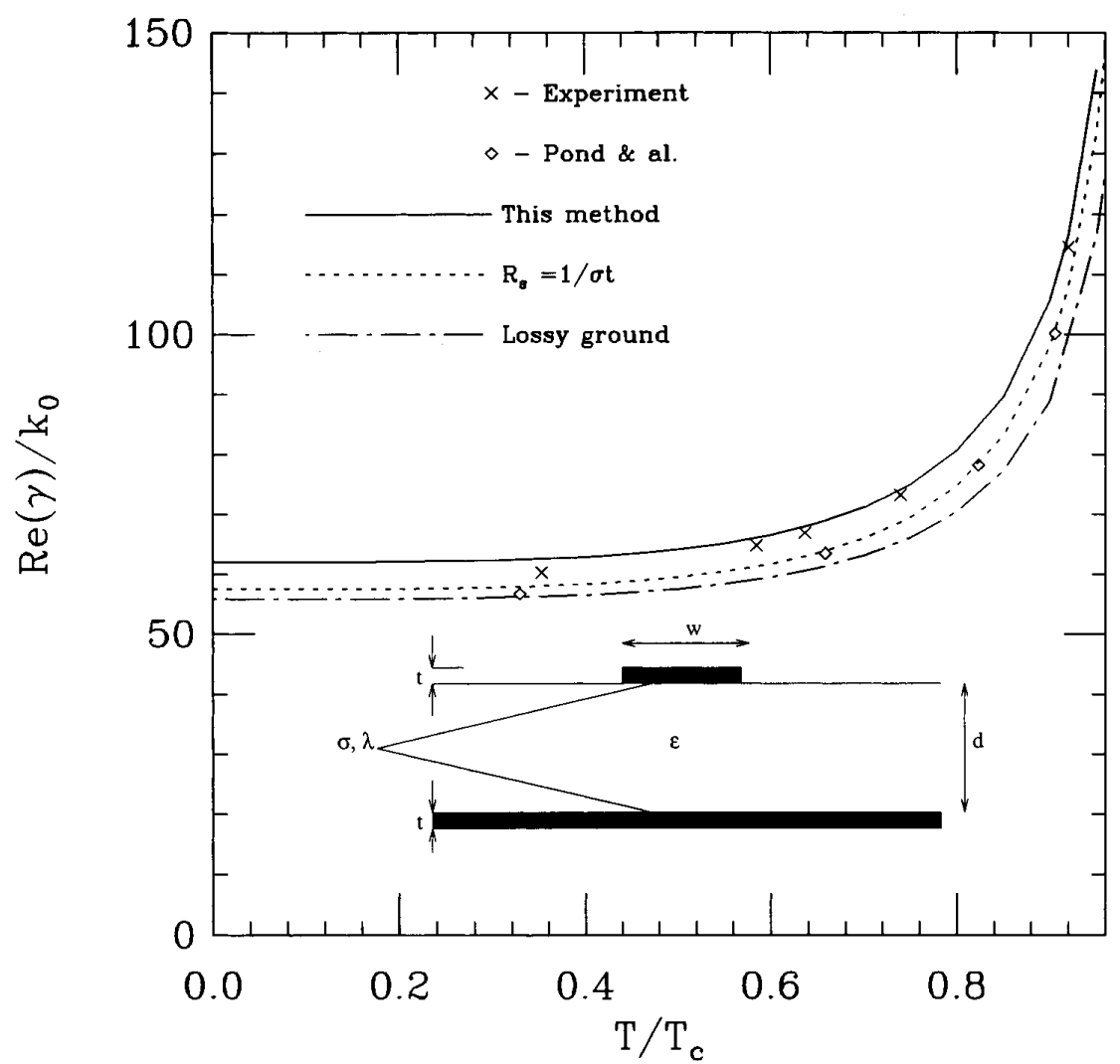

Figure 3. Real part of the propagation constant $\gamma / k_{0}$ as a function of $T / T_{\mathrm{c}}$ for a thin superconducting microstrip line at $1 \mathrm{GHz}$

The attenuation constant of the same structure is shown in Figure 6 as a function of $T / T_{\mathrm{c}}$ at $1 \mathrm{GHz}$. The attenuation constant increases monotonically with temperature, more precipitously at temperatures close to $T_{\mathrm{c}}$ or to 0 as expected from the variation of $\sigma_{1}=\sigma_{\mathrm{n}}\left(T / T_{\mathrm{c}}\right)^{4}$ with $T / T_{\mathrm{c}}$. The effect of the different LSE and LSM sheet impedances in our theory (solid line) is clearly visible.

The attenuation constant of a similar microstrip line, where the thickness of the substrate is increased to $4.5 \mu \mathrm{m}$, is shown in Figure 7. The solid line is from the present approach, where both diagonal as well as off-diagonal metallic elements are included. Note that, for comparison purposes, we also simulated the structure which has been used in Reference 1 instead of the actual microstrip line. The results obtained using $R_{\mathrm{s}}=1 / \sigma t$ coincide, within plotting accuracy, with those obtained from the present method.

Figure 8(a) shows the propagation constant as a function of $T / T_{\mathrm{c}}$ of the microstrip line of Figure 3 for different values of $I / I_{\mathrm{c}}$ at $1 \mathrm{GHz}$. It is interesting to note that the change in the normalized propagation constant is relatively small as long as the line is in the superconducting state. As expected, the system undergoes a phase transition at a temperature $T_{\mathrm{tr}}$, which decreases as the current in the line is increased. For this structure, the propagation constant in the normal state is larger than in the superconducting structure. This is mainly due to the considerable ohmic losses in the thin conductors. Indeed, when the resistance per unit length of a transmission line is much larger than its inductance, and the dielectric losses are small, the complex propagation constant is approximately given by $\gamma=(1+j) \sqrt{ }(R C \omega / 2)$. This observation also lends support to the approach presented here since it gives propagation and attenuation constants which are equal to within less than $5 \%$ in the normal state, i.e. $T>T_{\mathrm{c}}$.

The attenuation constant is, on the other hand, more sensitive to the transported current (power) as shown in Figure 8(b). Even at temperatures which are low compared to the transition temperature $T_{\text {tr }}$, the attenuation constant increases considerably faster than the propagation constant as the current in the line is increased. 


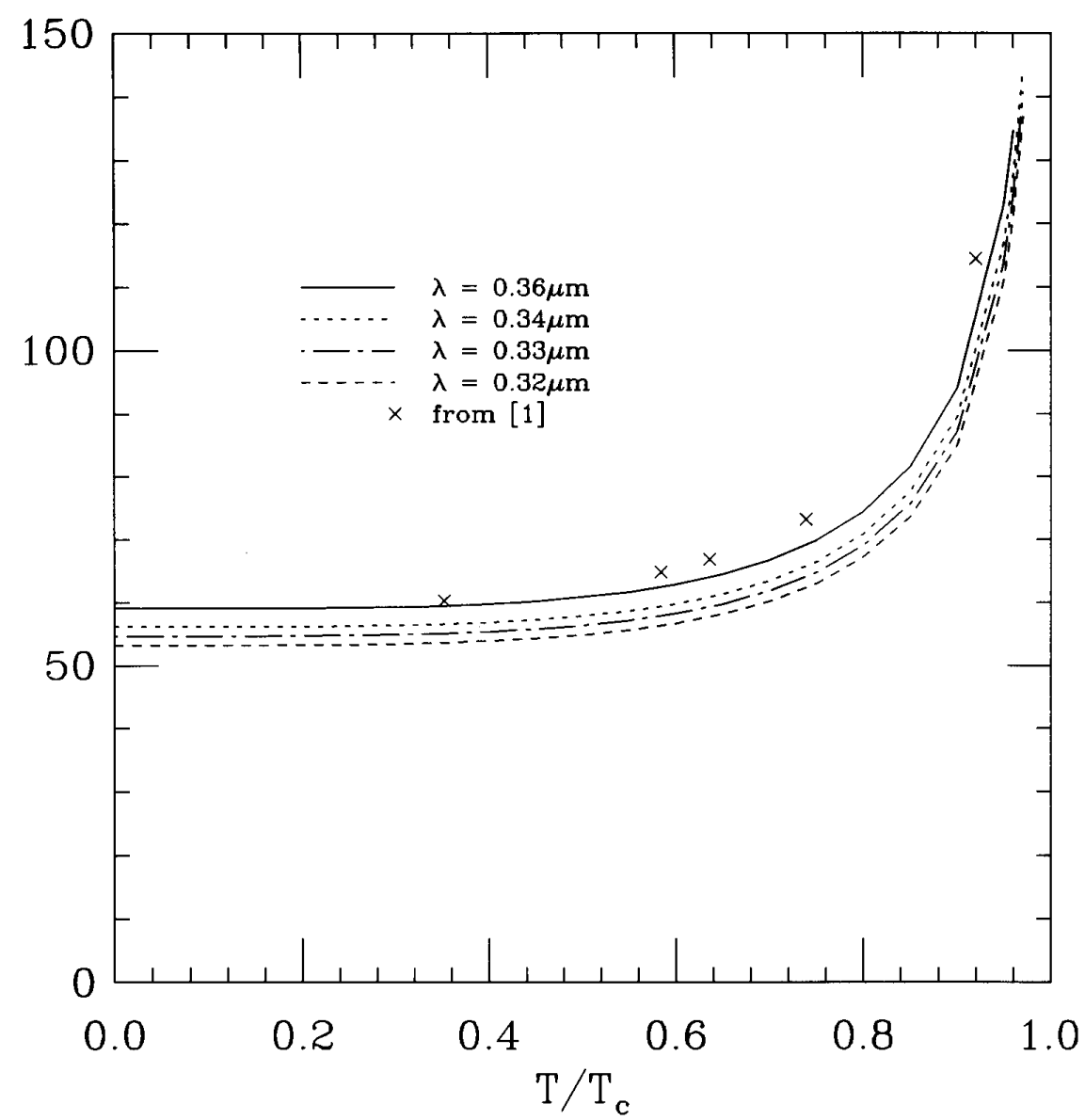

Figure 4. Propagation constant as a function of $T / T_{\mathrm{c}}$ of the microstrip in Figure 3 at $1 \mathrm{GHz}$ for different values of $\lambda_{L}$

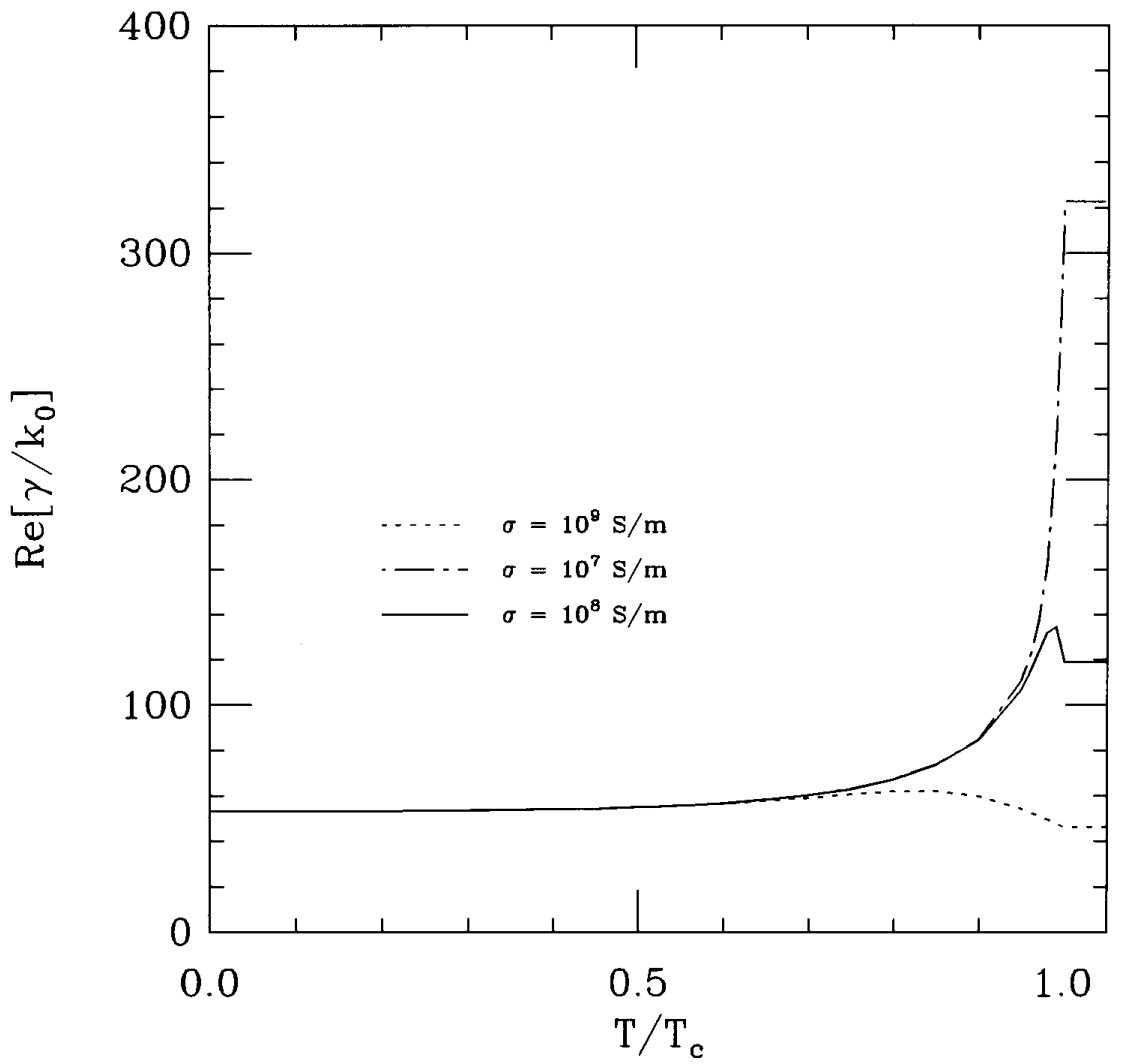

Figure 5. Propagation constant as a function of $T / T_{\mathrm{c}}$ of the microstrip in Figure 3 at $1 \mathrm{GHz}$ for different values of $\sigma_{n}$ 


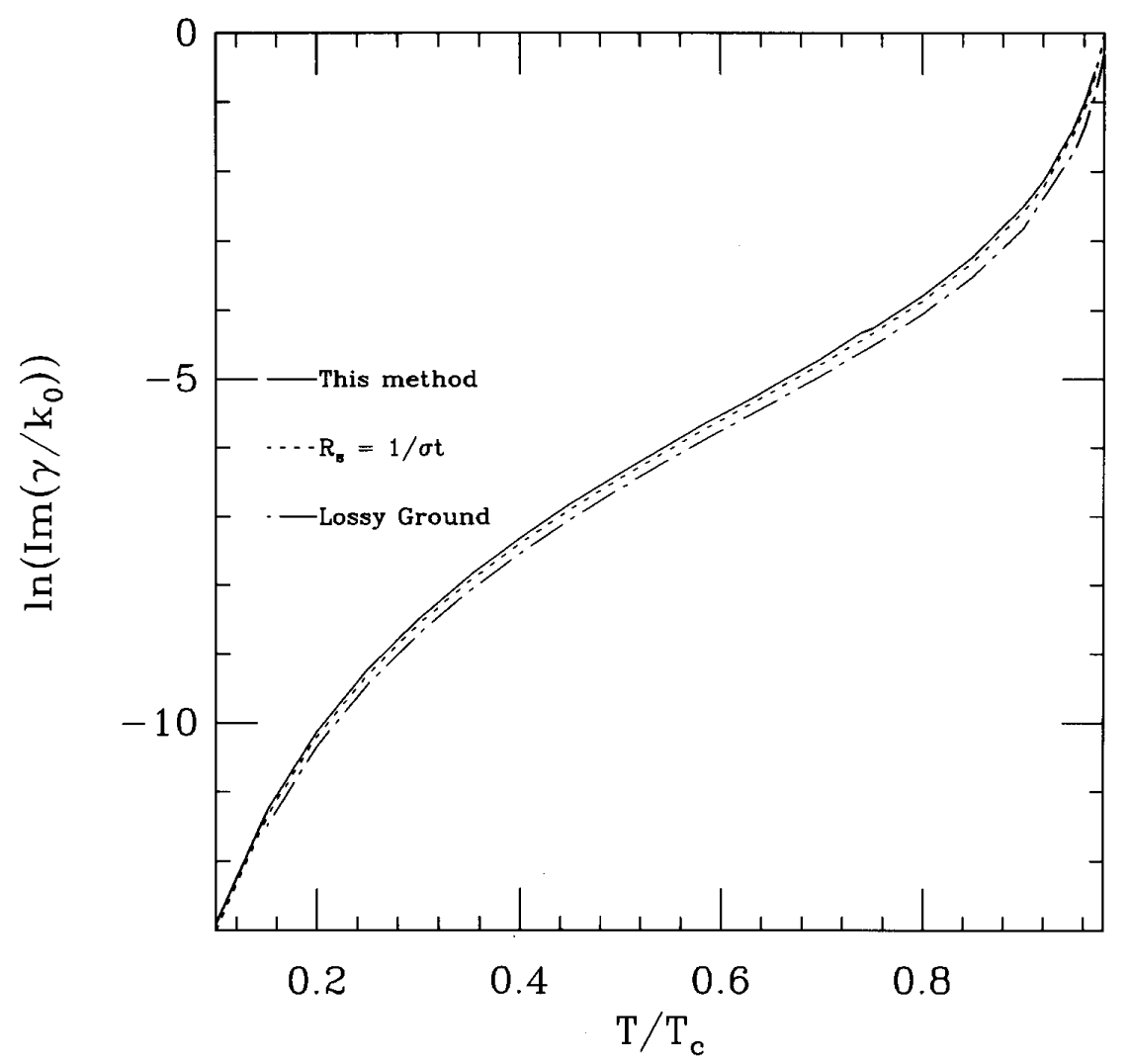

Figure 6. Attenuation constant $\ln \left[\alpha / k_{0}\right]$ as a function of $T / T_{\mathrm{c}}$ of the microstrip in Figure 3 at $1 \mathrm{GHz}$

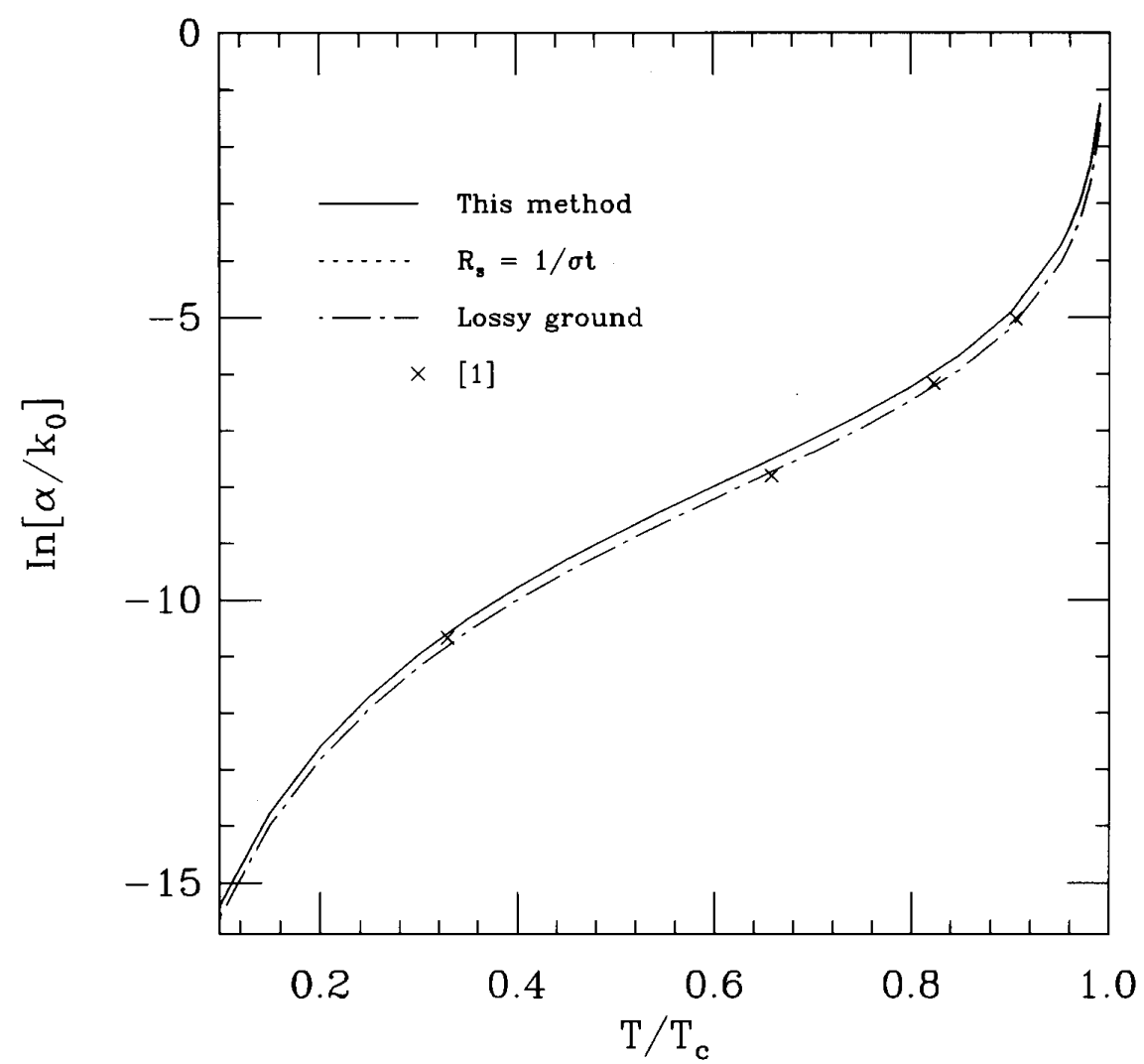

Figure 7. Attenuation constant of a microstrip line as a function of $T / T_{\mathrm{c}}$ at $1 \mathrm{GHz}$ and $d=4.5 \mu \mathrm{m}$. The crosses are from Reference 1 . Note that the solid and dashed lines are identical to within the plotting accuracy 


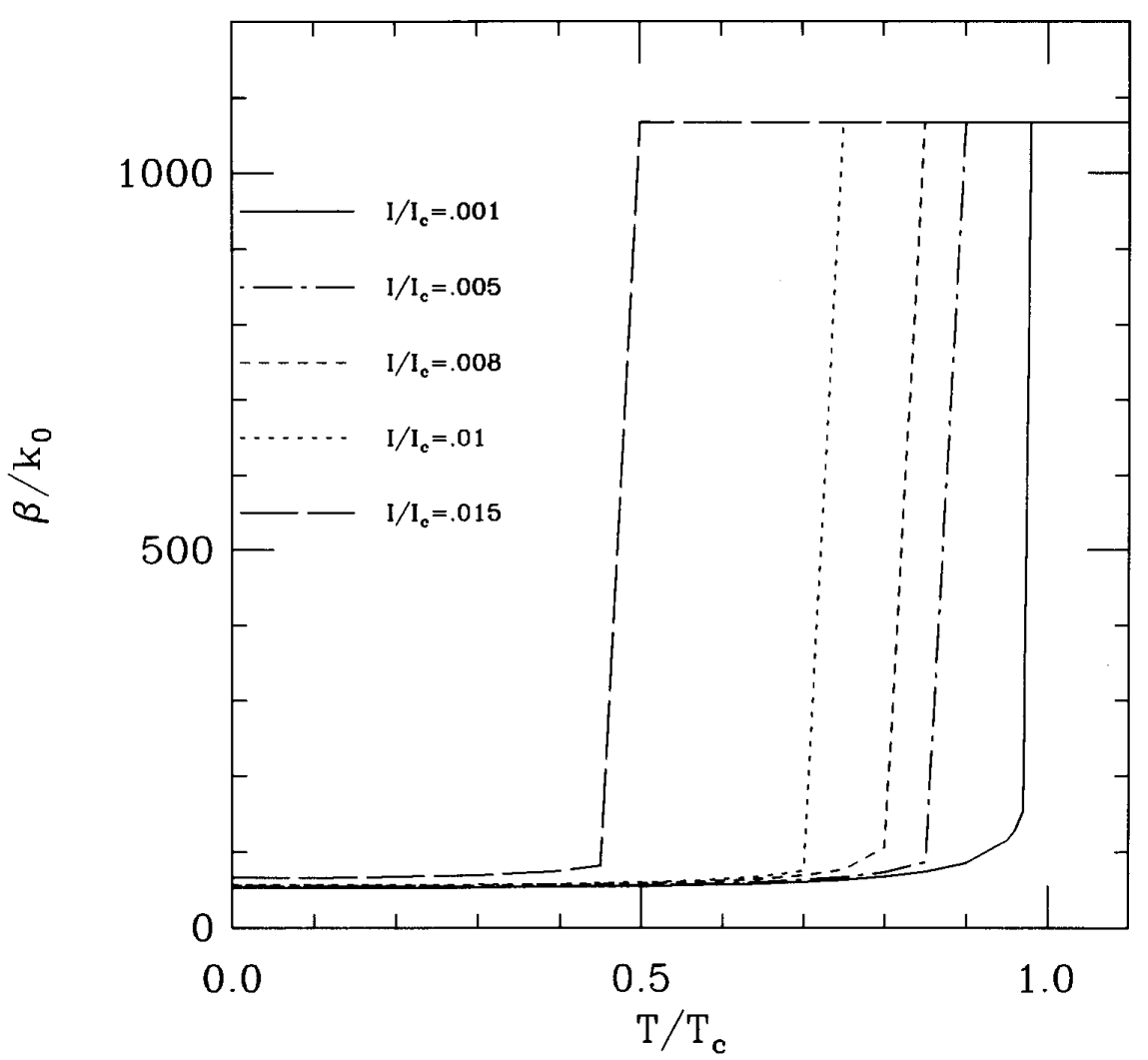

(a)

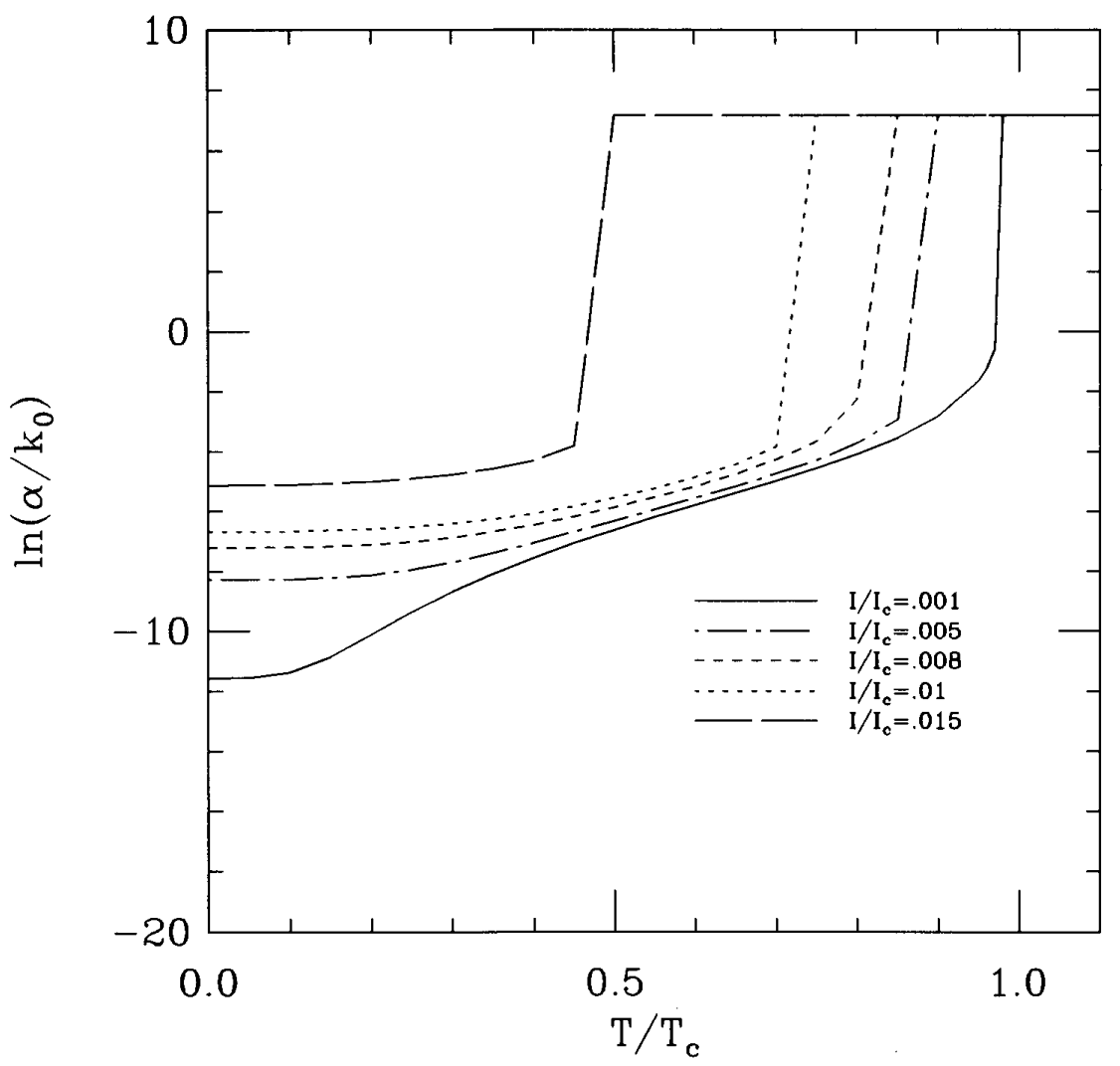

(b)

Figure 8. Propagation characteristics of the superconducting microstrip line of Figure 3 as a function of $T / T_{\mathrm{c}}$ at $1 \mathrm{GHz}$ for difference values of $I / I_{c}$ : (a) propagation constant; (b) attenuation constant 


\subsection{Patch resonator}

As a second example, our approach is applied to determine the resonant frequencies of a patch resonator (Figure 9(b)). Figure 9(a) shows the resonant frequency as a function of $T / T_{\mathrm{c}}$ for a square superconducting patch. The rapid increase in the resonant frequency, as the temperature is lowered below $T_{\mathrm{c}}$, is related to the increase in the propagation constant or, equivalently, the decrease in the phase velocity. The solid line corresponds to both diagonal and off-diagonal metallic elements, while the dashed line is obtained from only diagonal elements all equal to the conventional sheet resistance $1 / \sigma t$. The integrals appearing in the inner products were replaced by discrete summations, where $\alpha_{\mathrm{n}}=(n+0.5) \pi / a$ and $\beta_{\mathrm{n}}=m \pi / L, L=5 \mathrm{~mm}$ and $2 a=5 \mathrm{~mm}$. For the dimensions used, the difference between the two results is minor. This is due primarily to the

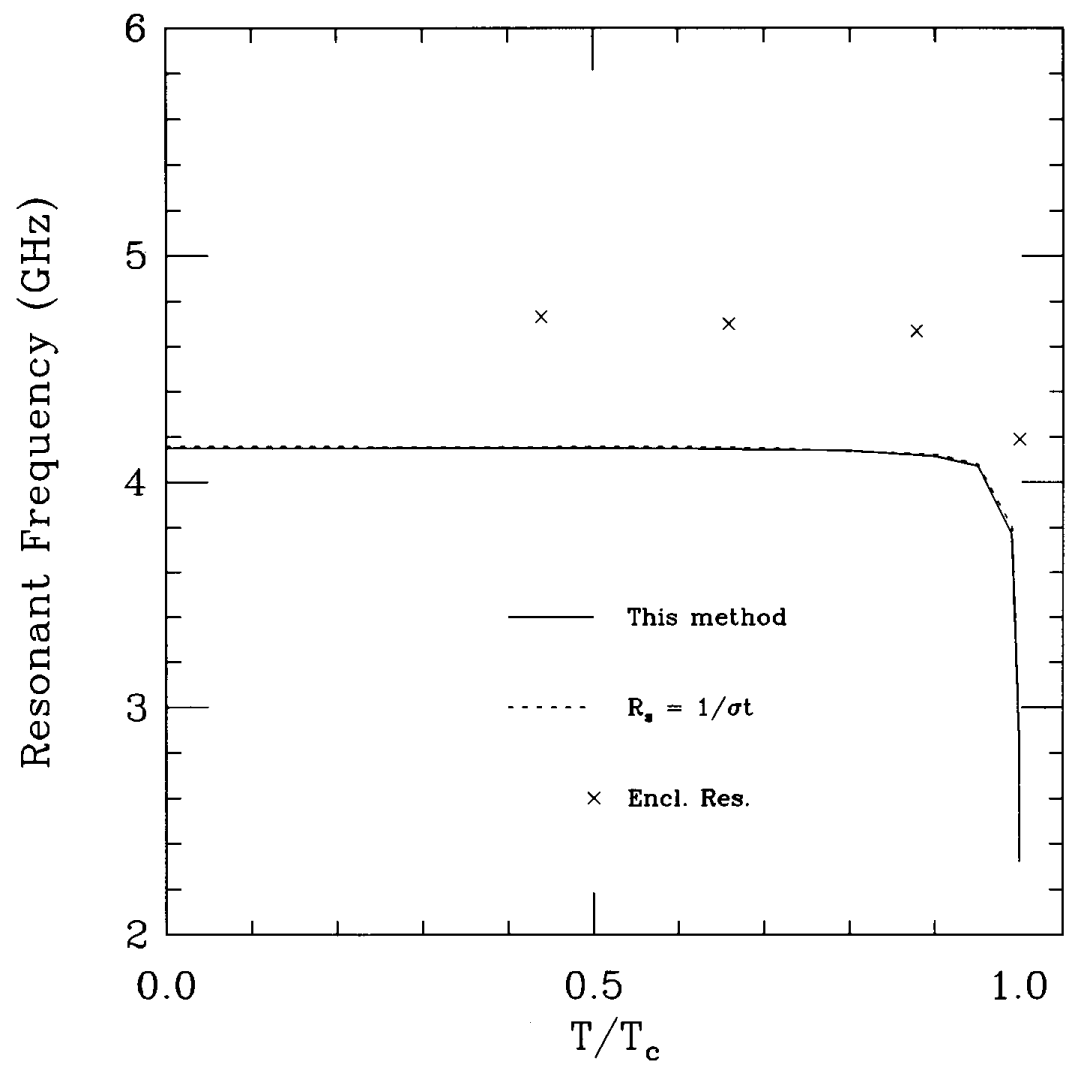

(a)

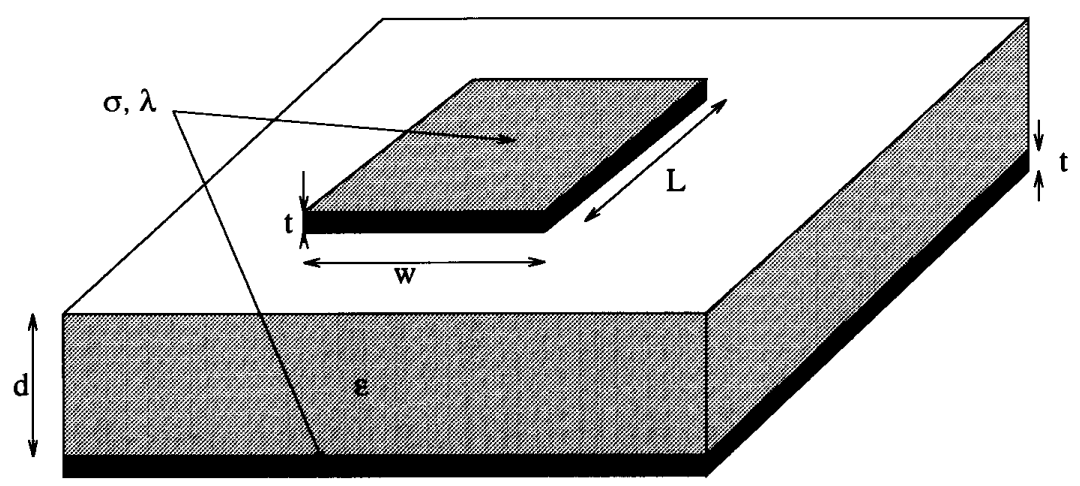

(b)

Figure 9. Resonant frequency (a) of a superconducting patch (b) as a function of $T / T_{\mathrm{c}} . \epsilon_{r}=24 \cdot 5, d=0.508 \mathrm{~mm}$, $\tan \delta=4 \times 10^{-5}, L=7.8 \mathrm{~mm}, W=0.5 \mathrm{~mm}, \lambda_{0}=0.68 \mu \mathrm{m}, \sigma_{n}=2 \times 10^{4} \mathrm{~S} / \mathrm{m}, t=\lambda$. The crosses are from Reference 18 for a boxed resonator with enclosure dimensions, $10 \times 5 \times 5.588 \mathrm{~mm}$ 
fact that larger values of $\alpha$ (or $\beta$ ), for which the LSE and LSM modes have significantly different sheet impedances, do not play a major role in describing a quantity which does not vary rapidly in space. The crosses, from Reference 18, are those of a boxed resonator with an upper shield of height $h=5.08 \mathrm{~mm}$. The enclosure confines the electromagnetic field to the volume of the box, thereby forcing more penetration in the superconductor. A higher inductance or resonant frequency results, as the positions of the crosses show.

\section{CONCLUSIONS}

A new approach to analysing superconducting microstrip structures is presented. The sheet impedance of a thin conductor is used to describe the response of the system to a current density. The Ginzburg-Landau equations were used to account for the effect of the magnetic field on the state of the superconductor. It is reported that the system undergoes a phase transition to the normal state at a temperature $T_{\text {tr }}$ which decreases as the current (power) transported is increased. The approach was applied to single microstrip line and a patch resonator. Good agreement was found with experimental results or previously published calculations.

\section{REFERENCES}

1. J. M. Pond, C. M. Krowne and W. L. Carter, 'On the application of complex resistive boundary conditions to mode transmission lines consisting of very thin superconductors', IEEE Trans. Microwave Theory Tech., 37, 181-190 (1989).

2. C. W. Kuo and T. Itoh, 'A flexible approach combining the spectral domain method and impedance boundary condition for the analysis of microstrip line', IEEE Microwave and Guided Wave Lett., 1, 172-174 (1991).

3. Z. Cai and J. Bornemann, 'Rigorous analysis of radiation properties of lossy patch resonators on complex anisotropic media and lossy ground metallization', IEEE Trans. Antennas Propag., 42, 1443-1446 (1994).

4. D. Nghiem, J. F. Williams and D. R. Jackson, 'A general analysis of propagation along multiple-layer superconducting stripline and microstrip transmission lines', IEEE Trans. Microwave Theory Tech., 39, 1553-1565 (1991).

5. S. Amari and J. Bornemann, 'LSE- and LSM-mode sheet impedances of thin conductors', IEEE Trans. Microwave Theory Tech., 44, 967-970 (1996).

6. R. E. Troxler, G. P. Rodrigue and P. N. Peters, 'Field response of ultra-thin type II superconducting transmission lines', IEEE Trans. Applied Superconductivity, 4, 65-75 (1994).

7. M. A. Megahed and S. M. El-Ghazaly, 'Full-wave nonlinear analysis of microwave superconductor devices: application to filters', IEEE MTT-S Int. Microwave Symp. Dig., 1265-1268 (May 1995).

8. J. W. Lynn, High Temperature Superconductivity, Springer-Verlag, New York, 1990.

9. D. H. Douglas, Jr., 'Magnetic field dependence of the superconducting penetration depth in thin specimens', Phys. Rev., 124, 735-739 (1961).

10. J. G. Ma and I. Wolff, 'Modeling the microwave properties of superconductors', IEEE Trans. Microwave Theory Tech., 43, 1053-1059 (1995).

11. K. Holczer et al., 'Observation of the conductivity coherence peak in superconducting $\mathrm{Bi}_{2} \mathrm{Sr}_{2} \mathrm{CaCu}_{2} \mathrm{O}_{8}$ single crystal', Phys. Rev. Lett., 67, 152-154 (1991).

12. S. M. El-Ghazaly, R. B. Hammond and T. Itoh, 'Analysis of superconducting microwave structures: Application to microstrip lines', IEEE Trans. Microwave Theory Tech., 40, 499-508 (1992).

13. M. Tinkham, Introduction to Superconductivity, McGraw-Hill, New York, 1975.

14. T. VanDuzer and C. W. Turner, Principles of Superconductive Devices and Circuits, Elsevier, New York, 1981.

15. T. P. Orlando and K. A. Delin, Foundations of Applied Superconductivity, Addison-Wesley, New York, 1991.

16. S. Amari and J. Bornemann, 'Non-perturbative fullwave analysis of lossy planar circuits', IEEE MTT-S Int. Microwave Symp. Dig., 1277-1280 (May 1995).

17. J. C. Swihart, 'Field solution for a thin-film superconducting strip transmission line', J. Appl. Phys., 32, 461-469 (1961).

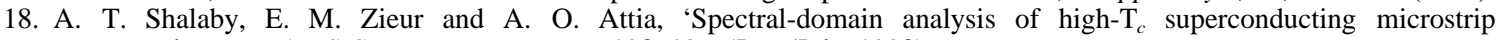
resonators', IEEE AP-S Symposium Dig., pp. 193-196 (June/July 1993).

Authors' biographies:

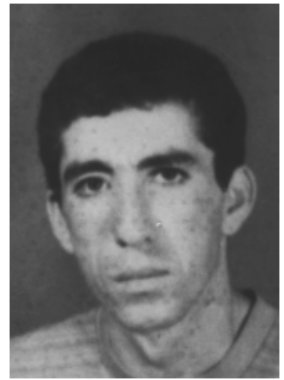

Smain Amari received his D.E.S. in Physics and Electronics from Constantine University (Algeria) in 1985, the M.S. degree in Electrical Engineering in 1989 and the Ph.D. degree in Physics in 1994, both from Washington University in St. Louis. He is interested in numerical methods in electromagnetics, numerical analysis, applied mathematics, applied physics and application of quantum field theory in quantum many-particle systems. Since 1994, he has been with the Department of Electrical and Computer Engineering at the University of Victoria, Canada. 


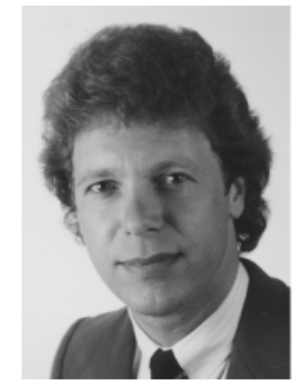

Jens Bornemann was born in Hamburg, Germany, on 26 May 1952. He received the Dipl.-Ing. and the Dr.-Ing. degrees, both in Electrical Engineering, from the University of Bremen, Germany, in 1980 and 1984, respectively.

From 1980 to 1983, he was a Research and Teaching Assistant in the Microwave Department at the University of Bremen, where he worked on quasi-planar waveguide configurations and computer-aided E-plane filter design. In 1985, after a two-year period as a Consulting Engineer, he joined the University of Bremen again and was employed at the level of Assistant Professor. Since April 1988, he has been with the University of Victoria, Victoria, B.C., Canada, where he is currently a Professor in the Department of Electrical and Computer Engineering. His research activities include microwave/millimetre-wave components and systems design, and problems of electromagnetic field theory in integrated circuits and radiating structures. He is a coauthor of Waveguide Components for Antenna Feed Systems. Theory and Design (Artech House, 1993) and has authored/coauthored more than 100 technical papers. He is a Registered Professional Engineer in the Province of British Columbia, Canada, and has been a Fellow of the British Columbia Advanced Systems Institute, 1992-1995.

Dr. Bornemann was one of the recipients of the A.F. Bulgin Premium of the Institution of Electronic and Radio Engineers in 1983. He is a Senior Member of the IEEE and serves on the editorial boards of the IEEE Transactions on Microwave Theory and Techniques and the International Journal of Numerical Modelling. 